\title{
The Effect of Field Changing on Two Qubit Entanglement with Different Criteria
}

\author{
M. MirzaeE* AND M. BatAvani \\ Department of Physics, Faculty of Science, Arak University, Arak 38156-8-8349, Iran \\ (Received February 9, 2014; revised version December 14, 2014; in final form December 17, 2014) \\ We investigate the effect of field changing on two qubit entanglement. We choose the coherent state, the \\ cat state and the squeezed state as different states of interacting field with atom. The correlation between two \\ qubits can be degraded by environmental noise, this phenomenon has been labelled sudden death of entanglement. \\ The double Jaynes-Cummings model as a solvable model is used for describing the dynamic of sudden death \\ of entanglement. We measure two-qubit entanglement using the concurrence and the negativity. The Fisher \\ information is introduced, too, and compares results with previous entanglement measures. Finally we conclude \\ that the Fisher information is not an entanglement measure.
}

DOI: 10.12693/APhysPolA.127.663

PACS: 03.65.Ud

\section{Introduction}

Quantum entanglement plays an important role in many quantum information and quantum computation tasks [1-7]. Recently, the Jaynes-Cummings model (JCM) has been noticed, JCM describes the interaction between a single-mode quantized field and a single twolevel atom [8]. On the other hand, since the quantum system interacts with background or environment, we expect that its entanglement falls. It has been shown that entanglement can vanish to zero suddenly in a finite time, this phenomenon is called entanglement sudden death (ESD) [9-17]. Qian and Eberly showed that the rules restricting the occurrence and timing of ESD, depend on only three parameters: initial purity, entanglement and excitation so that knowledge or control of initial phases is not needed [18]. Yönaç and Eberly showed that error correction affects entanglement and fidelity differently, and also entangled states for which error correction increases fidelity but decreases entanglement, and vice versa [19]. Hoang showed geometric invariants provide useful information about different types of entanglement based on double JCM [20]. Zhang and Xie showed that the larger the decay coefficient is the more quickly the entanglement between two atoms in a JCM decays [21]. Oliveira et al. showed that whenever entanglement sudden death occurs in one of the partitions residual entanglement will appear [22]. Effect of light squeezing on the entanglement dynamics in JCM was investigated by Subeesh et al. They concluded that the atom has a tendency to get disentangled from field within the collapse region and also in the revival region, for mild squeezing [23].

In this paper, we investigate ESD of two two-level atoms. At first, we select the coherent state as an interacting field with qubit and measure two-qubit entan-

${ }^{*}$ corresponding author; e-mail: m-mirzaee@araku.ac.ir glement using concurrence and negativity. On the other hand, quantum Fisher information is used for studying some properties of quantum states. Although some authors have introduced the quantum Fisher information as a parameter of entanglement, it is not clear that this function can detect the quantum behaviour.

Thereafter we change interacting field from coherent state to the cat state and repeat the measurements of entanglement using introduced criteria. Finally we choose the squeezed state and measure the qubit entanglement. We have selected the double JCM consisting of two twolevel atoms. Each one is interacting with its cavity mode and has a ground state $|g\rangle$ and an excited state $|e\rangle$. Each field (cavity mode) is supposed to be exactly resonant. Each cavity-atom is completely isolated from the other atom and cavity. The double Jaynes-Cummings Hamiltonian for our system can be written as

$$
\begin{gathered}
H_{\mathrm{Tot}}=\omega \sigma_{z}^{A}+\omega \sigma_{z}^{b}+g\left(a^{\dagger} \sigma_{-}^{A}+a \sigma_{+}^{A}\right) \\
+g\left(a^{\dagger} \sigma_{-}^{B}+a \sigma_{+}^{B}\right)+\nu a^{\dagger} a+\nu b^{\dagger} b,
\end{gathered}
$$

where $\omega$ is the atomic transition frequency; $\nu$ is the frequency of the cavity field ( $\omega=\nu$ resonance); $g$ is the atom-field coupling constant; $a(b)$ and $a^{\dagger}\left(b^{\dagger}\right)$ are the field annihilation and creation operators; and $\sigma_{ \pm}$are the atomic "spin-flip" operators.

\section{Time evolution of the system}

In Fig. 1 for a partially entangled atomic pure state that is a combination of the Bell states we consider two different atomic initial states as follows:

$$
\begin{aligned}
& \left|\psi_{a}(0)\right\rangle=\cos (\beta)|e g\rangle+\sin (\beta)|g e\rangle, \\
& \left|\psi_{a}(0)\right\rangle=\cos (\beta)|e e\rangle+\sin (\beta)|g g\rangle,
\end{aligned}
$$

where $\left|\psi_{a}(0)\right\rangle=\left|\psi_{\text {atom }}(0)\right\rangle$. First off, we assume that the field is initially in coherent state $\left|\psi_{\mathrm{F}}(0)\right\rangle=|\alpha\rangle=$

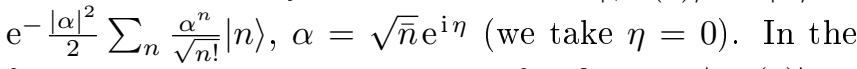
following we choose a cat state for field as $\left|\psi_{\mathrm{F}}(0)\right\rangle=$ $|c\rangle=N\left(|\alpha\rangle+\mathrm{e}^{\mathrm{i} \varphi}|-\alpha\rangle, \quad N=\sqrt{\left(2+2 \mathrm{e}^{-2 \alpha^{2}} \cos (\varphi)\right)}\right.$ 
(we take $\phi=0$ ) [24]. The last election for field is the squeezed coherent state, $\left|\psi_{\mathrm{F}}(0)\right\rangle=|s\rangle=s(\lambda)|\alpha\rangle$, where $s(\lambda)$ is the single mode squeezing operator given by $s(\lambda)=\exp \left(\frac{1}{2}\left(\lambda^{*} a^{2}-\lambda a^{\dagger 2}\right)\right), \lambda$ is a squeezing parameter $\lambda=r \mathrm{e}^{\mathrm{i} \theta}$ (we take $\theta=0$ ) [24]. Under these conditions we can consider six initial states for system in Fig. 1 and then investigate the time evolution of qubit entanglement for six initial wave function of the total system that are given by

$$
\begin{aligned}
\left|\psi_{1}(0)\right\rangle & =\cos (\beta)|e g \alpha \alpha\rangle+\sin (\beta)|g e \alpha \alpha\rangle, \\
\left|\psi_{2}(0)\right\rangle & =\cos (\beta)|e e \alpha \alpha\rangle+\sin (\beta)|g g \alpha \alpha\rangle, \\
\left|\psi_{3}(0)\right\rangle & =\cos (\beta)|e g c c\rangle+\sin (\beta)|g e c c\rangle, \\
\left|\psi_{4}(0)\right\rangle & =\cos (\beta)|e e c c\rangle+\sin (\beta)|g g c c\rangle, \\
\left|\psi_{5}(0)\right\rangle & =\cos (\beta)|e g s s\rangle+\sin (\beta)|g e s s\rangle, \\
\left|\psi_{6}(0)\right\rangle & =\cos (\beta)|e e s s\rangle+\sin (\beta)|g g s s\rangle
\end{aligned}
$$

where the first and second indexes denote the state of qubit atomic and the others introduce the fields. It can be shown that the wave function of the total system $|\psi(t)\rangle$ under the Hamiltonian (1) evolves in the standard way and take a doubly infinite series summation as

$$
\begin{aligned}
& |\psi(t)\rangle=\sum_{n, m}\left[a_{n, m}(t) \mid \text { ee } n, m\right\rangle+b_{n, m}(t)|\operatorname{eg} n, m\rangle \\
& \left.\left.\quad+c_{n, m}(t) \mid \text { ge } n, m\right\rangle+d_{n, m}(t)|g g n, m\rangle\right] .
\end{aligned}
$$

After using the Schrödinger equation for the initial state (4), we get the coefficients as

$$
\begin{gathered}
a_{n, m}(t)= \\
-\mathrm{i} A\left(\frac{\alpha}{\sqrt{m+1}} \cos (\beta) C_{1 n} S_{1 m}+\frac{\alpha}{\sqrt{n+1}} \sin (\beta) S_{1 n} C_{1 m}\right), \\
b_{n, m}(t)=A\left(\cos (\beta) C_{1 n} C_{0 m}-\frac{\sqrt{m}}{\sqrt{n+1}} \sin (\beta) S_{1 n} S_{0 m}\right), \\
c_{n, m}(t)=A\left(\sin (\beta) C_{0 n} C_{1 m}-\frac{\sqrt{n}}{\sqrt{m+1}} \sin (\beta) S_{1 m} S_{0 n}\right), \\
d_{n, m}(t)= \\
-\mathrm{i} A\left(\frac{\sqrt{n}}{\alpha} \cos (\beta) C_{0 m} S_{0 n}-\frac{\sqrt{m}}{\alpha} \sin (\beta) S_{0 m} C_{0 n}\right),
\end{gathered}
$$

where

$$
\begin{aligned}
& A=\mathrm{e}^{-|\alpha|^{2}} \frac{|\alpha|^{n+m}}{\sqrt{n ! m !}}, \\
& C_{1 n(m)}=\cos (g t \sqrt{n(m)+1}), \\
& C_{0 n(m)}=\cos (g t \sqrt{n(m)}), \\
& S_{1 n(m)}=\sin (g t \sqrt{n(m)+1}), \\
& S_{0 n(m)}=\sin (g t \sqrt{n(m)}) .
\end{aligned}
$$

Similarly with changing the initial state from (4) to (5) the coefficients convert to:

$$
\begin{aligned}
& a_{n, m}(t)= \\
& A\left(\cos (\beta) C_{1 n} C_{1 m}-\frac{\alpha^{2}}{\sqrt{(n+1)(m+1)}} \sin (\beta) S_{1 n} S_{1 m}\right),
\end{aligned}
$$

$$
\begin{aligned}
& \quad b_{n, m}(t)= \\
& -\mathrm{i} A\left(\frac{\sqrt{m}}{\alpha} \cos (\beta) C_{1 n} S_{0 m}+\frac{\alpha}{\sqrt{n+1}} \sin (\beta) S_{1 n} C_{0 m}\right), \\
& \quad c_{n, m}(t)= \\
& -\mathrm{i} A\left(\frac{\sqrt{n}}{\alpha} \sin (\beta) S_{0 n} C_{1 m}+\frac{\sqrt{\alpha}}{\sqrt{m+1}} \sin (\beta) S_{1 m} C_{0 n}\right), \\
& \quad d_{n, m}(t)= \\
& A\left(-\frac{\sqrt{n m}}{\alpha^{2}} \cos (\beta) S_{0 m} S_{0 n}+\sin (\beta) C_{0 m} C_{0 n}\right) .
\end{aligned}
$$

We can solve Schrödinger equation with initial state (6), too, and calculate the coefficients as

$$
\begin{aligned}
& a_{n, m}(t)=-\mathrm{i} A\left(\frac{\alpha}{\sqrt{m+1}} \chi_{1 n} \chi_{2 m} \cos (\beta) C_{1 n} S_{1 m}\right. \\
& \left.+\chi_{1 m} \chi_{2 n} \frac{\alpha}{\sqrt{n+1}} \sin (\beta) S_{1 n} C_{1 m}\right), \\
& b_{n, m}(t)=A\left(\chi_{1 n} \chi_{1 m} \cos (\beta) C_{1 n} C_{0 m}\right. \\
& \left.-\frac{\sqrt{m}}{\sqrt{n+1}} \chi_{2 n} \chi_{2 m} \sin (\beta) S_{1 n} S_{0 m}\right), \\
& c_{n, m}(t)=A\left(\chi_{1 n} \chi_{1 m} \sin (\beta) C_{0 n} C_{1 m}\right. \\
& \left.-\frac{\sqrt{n}}{\sqrt{m+1}} \chi_{2 n} \chi_{2 m} \sin (\beta) S_{1 m} S_{0 n}\right), \\
& d_{n, m}(t)=-\mathrm{i} A\left(\frac{\sqrt{n}}{\alpha} \chi_{1 m} \chi_{2 n} \cos (\beta) C_{0 m} S_{0 n}\right. \\
& \left.-\frac{\sqrt{m}}{\alpha} \chi_{1 n} \chi_{2 m} \sin (\beta) S_{0 m} C_{0 n}\right),
\end{aligned}
$$

where $\chi_{1 n(m)}=1+(-1)^{n(m)}$ and $\chi_{2 n(m)}=1-(-1)^{n(m)}$. Also for the initial state $(7)$ we have the following terms for coefficients:

$$
\begin{aligned}
& a_{n, m}(t)=A\left(\chi_{1 n} \chi_{1 m} \cos (\beta) C_{1 n} C_{1 m}\right. \\
& \left.-\frac{\alpha^{2}}{\sqrt{(n+1)(m+1)}} \chi_{2 n} \chi_{2 m} \sin (\beta) S_{1 n} S_{1 m}\right), \\
& b_{n, m}(t)=-\mathrm{i} A\left(\frac{\sqrt{m}}{\alpha} \chi_{1 n} \chi_{2 m} \cos (\beta) C_{1 n} S_{0 m}\right. \\
& \left.+\frac{\alpha}{\sqrt{n+1}} \chi_{1 m} \chi_{2 n} \sin (\beta) S_{1 n} C_{0 m}\right), \\
& c_{n, m}(t)=-\mathrm{i} A\left(\frac{\sqrt{n}}{\alpha} \chi_{1 n} \chi_{2 m} \sin (\beta) S_{0 n} C_{1 m}\right. \\
& \left.+\frac{\sqrt{\alpha}}{\sqrt{m+1}} \chi_{1 m} \chi_{2 n} \sin (\beta) S_{1 m} C_{0 n}\right), \\
& d_{n, m}(t)=A\left(-\frac{\sqrt{n m}}{\alpha^{2}} \chi_{2 n} \chi_{2 m} \cos (\beta) S_{0 m} S_{0 n}\right. \\
& \left.+\chi_{1 n} \chi_{1 m} \sin (\beta) \cos (g t \sqrt{m}) \cos (g t \sqrt{n})\right) .
\end{aligned}
$$

Similarly we obtain coefficients for primary states (8) and (9), these coefficients are very long, so we avoid writing them.

All information is contained in density matrix of system that can be obtained from wave function (10) as a 


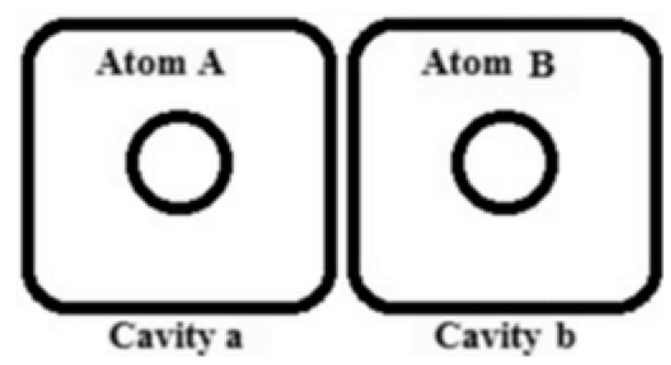

Fig. 1. The picture of the two atoms in two cavity interacting with different fields.

pure state $\rho(t)=|\psi(t)\rangle\langle\psi(t)|$. Of course noting the semiclassical nature of the coherent state field we expect that its quantum qualities is not dominant, thus for measuring the qubit entanglement, we will trace out over the field of density matrix.

\section{Concurrence}

In this section we will use Wootters' concurrence [25] as our entanglement measure which is given by

$$
C(\rho)=\max \left(0, \sqrt{\lambda_{1}}-\sqrt{\lambda_{2}}-\sqrt{\lambda_{3}}-\sqrt{\lambda_{4}}\right),
$$

where the quantities $\lambda_{i}$ are the eigenvalues in decreasing order of the matrix $\tilde{\rho}$ where

$$
\tilde{\rho}=\rho\left(\sigma_{y} \bigotimes \sigma_{y}\right) \rho^{*}\left(\sigma_{y} \bigotimes \sigma_{y}\right) \text {. }
$$

Here $\rho$ is our two-qubit reduced state density matrix, $\rho^{*}$ denotes the complex conjugation of $\rho$ in the standard basis and $\sigma$ is the Pauli matrix. Using the wave function (10), Eq. (15) turns into

$$
\begin{aligned}
C & =\max \left(0 \sum_{n, m}\left|a_{n, m}(t)\right|^{2}\left|d_{n, m}(t)\right|^{2}\right. \\
& -b_{n, m}(t)^{*} c_{n, m}(t)^{*} a_{n, m}(t) d_{n, m}(t) \\
& -a_{n, m}(t)^{*} d_{n, m}(t)^{*} b_{n, m}(t) c_{n, m}(t) \\
& \left.+\left|b_{n, m}(t)\right|^{2}\left|c_{n, m}(t)\right|^{2}\right),
\end{aligned}
$$

where coefficients $(a, b, c, d)$ have been given in the previous section. Since in Eq. (17) there are $\cos (g t \sqrt{n})$ and $\sin (g t \sqrt{m})$, we expect to see similar revival behavior here. Figure 2 shows the time evolution of concurrence for six different initial system states, we note that the all curves in Fig. 2 indicate repeated occurrences of early-stage decoherence (ESD). We see an interesting result in Fig. 2a: the initial functions (4) and (5) create identical entanglement. This also applies in the case of Fig. $2 \mathrm{~b}$ and Fig. 2c. Figure 2b shows the concurrence changes of initial functions (6) and (7) and Fig. 2c relates to functions (8) and (9).

Comparing Fig. 2a and Fig. 2b, we see that Fig. 2b shows obviously more number of oscillations, this means the effect of field changing from the coherent state to cat state, is noticeable on the qubit entanglement, such that in Fig. 2b decay of the qubit entanglement is smoother than the coherent state field. In Fig. 2a the entanglement falls suddenly to zero and will remain zero for a period of time before entanglement recovers. In Fig. $2 \mathrm{~b}$ and $\mathrm{c}$ the

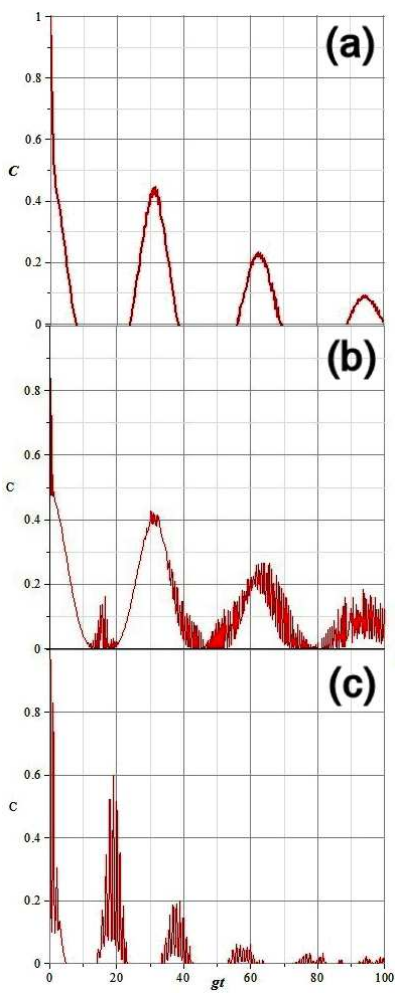

Fig. 2. The time evolution of the concurrence for initial functions (a) (4) and (5), (b) (6) and (7),(c) (8) and (9) with $\lambda=0.5$.

length of the time interval for the zero entanglement is too short, as soon as the entanglement falls, it recovers immediately. On the other hand, in Fig. 2c the entanglement is maximized five times $(t=0,21,42,64,86)$ while in the other diagrams this maximizing occurs by four times. According to the concurrence criterion, if the interacting field of qubit is in the coherent state, decrease of entanglement will occur rapidly in comparison with the other field states (cat and squeezed states).

\section{Negativity}

The Peres-Horodecki criterion for separability [26, 27] leads to natural computable measure of entanglement called negativity [28]. The negativity is based on the trace norm of the partial transposition $\rho^{T_{1}}$ of the bipartite mixed state $\rho$ and measures the degree to which $\rho^{T_{1}}$ fails to be positive, i.e., the absolute value of the sum of the negative eigenvalues of $\rho^{T_{1}}, N=\frac{\left\|\rho^{T_{1}}\right\|-1}{2}$, where $\left\|\rho^{T_{1}}\right\|$ denotes the trace norm of $\rho^{T_{1}}\left(\left\|\rho^{T_{1}}\right\|=\operatorname{Tr} \sqrt{\rho \rho^{\dagger}}\right)$.

Briefly, we can write the negativity as [29]:

$$
N=2 \max \left(0,-\lambda_{\min }\right) \text {, }
$$

where $\lambda_{\min }$ is the lowest eigenvalue of the partial transposition of the state $\rho^{T_{1}}$. It can be shown that using the wave function (10), the negativity converts to

$$
N=\sum_{n, m}\left(a_{n, m}(t) d_{n, m}(t)+b_{n, m}(t) c_{n, m}(t)\right) .
$$

Equation (19) contains of sine and cosine terms so except 


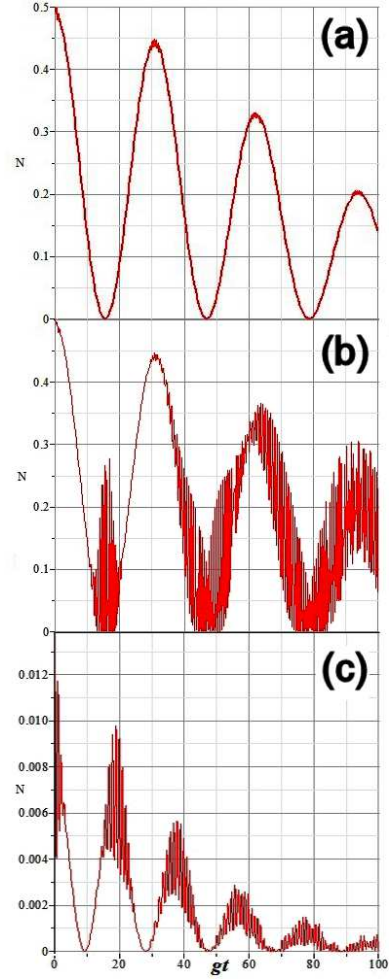

Fig. 3. The time evolution of the negativity for initial functions (a) (4) and (5), (b) (6) and (7), (c) (8) and (9) with $\lambda=0.5$.

to negativity exhibits an oscillating behavior. Figure 3 shows the behavior of the negativity with respect to time.

According to the negativity, the initial states (4) $((6),(8))$ and $(5)((7),(9))$ have created identical entanglement. Figure 3 a related to initial states (4) and (5), Fig. 3b to (6) and (7), Fig. 3c to initial functions (8) and (9). Unlike Fig. 2, in all curves of Fig. 3 entanglement suddenly falls and improves immediately, so that the length of time interval for the zero entanglement is approximately zero. Curves in Fig. 3c show more oscillations with respect to other curves. However, in comparing Fig. 2 we see that negativity is a stronger indicator than concurrence, because it could detect entanglement where concurrence had distinguished that the system is separable.

\section{Fisher information}

The Fisher information (FI) was devised by Fisher [30] and has recently been used as a parameter of entanglement [31-33]. The atomic Fisher information FI is defined as [32]:

$$
\begin{aligned}
\mathrm{FI} & =\sum_{\substack{j=1\\
}}^{2} \int_{0}^{2 \pi} \int_{0}^{\pi} Q_{A}(\theta, \varphi, t)\left(\sigma_{j} \frac{\partial \ln Q_{A}(\theta, \varphi, t)}{\partial \vartheta_{j}}\right)^{2} \\
& \times \sin (\theta) \mathrm{d} \theta \mathrm{d} \varphi,
\end{aligned}
$$

where $\left(\vartheta_{1}, \vartheta_{2}\right)=(\theta, \varphi), \sigma_{j}^{2}=\left\langle\vartheta_{j}^{2}\right\rangle-\left\langle\vartheta_{j}\right\rangle^{2}$ and $Q_{A}(\theta, \varphi, t)$ is the atomic Q-function given by $Q_{A}(\theta, \varphi, t)=$ $\frac{1}{2 \pi}\left\langle\theta, \varphi\left|\rho_{A}(t)\right| \theta, \varphi\right\rangle,|\theta, \varphi\rangle$ is the atomic coherent state that for two-level atom is written as $|\theta, \varphi\rangle=\cos \left(\frac{\theta}{2}\right)|e\rangle+$ $\sin \left(\frac{\theta}{2}\right) \exp (\mathrm{i} \varphi)|g\rangle$. By selecting the same parameters for the atomic coherent states of both systems in Fig. 1 as $\left(\theta_{A}=\theta_{B}\right.$ and $\left.\varphi_{A}=\varphi_{B}=0\right)$ the atomic coherent state of two qubit can be written as:

$$
\begin{aligned}
& |\theta, \varphi\rangle=\cos ^{2}\left(\frac{\theta}{2}\right)|e e\rangle+\frac{1}{2} \sin (\theta)|e g\rangle+\frac{1}{2} \sin (\theta)|g e\rangle \\
& \quad+\sin ^{2}\left(\frac{\theta}{2}\right)|g g\rangle .
\end{aligned}
$$

Now using Eq. (21) and the wave function (10) we can obtain the following formula for $Q$-function of qubit at $t>0$ :

$$
\begin{aligned}
& Q(t)=\sum_{n, m}\left(\cos \left(\frac{\theta}{2}\right)^{4}\left|a_{n, m}(t)\right|^{2}\right. \\
+ & \frac{1}{2} \sin (\theta)\left[\operatorname { c o s } ( \frac { \theta } { 2 } ) ^ { 2 } \left(a_{n, m}(t) b_{n, m}(t)^{*}+b_{n, m}(t) a_{n, m}(t)^{*}\right.\right. \\
+ & \left.a_{n, m}(t) c_{n, m}(t)^{*}+c_{n, m}(t) a_{n, m}(t)^{*}\right) \\
+ & \sin (t)^{2}\left(d_{n, m}(t) c_{n, m}(t)^{*}+c_{n, m}(t) d_{n, m}(t)^{*}\right) \\
& \left.\frac{1}{2}\left(a_{n, m}(t) d_{n, m}(t)^{*}+d_{n, m}(t) a_{n, m}(t)^{*}\right)\right] \\
+ & \left.\frac{1}{4} \sin (t)^{2}\left[\left|b_{n, m}(t)+c_{n, m}(t)\right|^{2}\right]+\sin \left(\frac{\theta}{2}\right)^{4}\left|d_{n, m}(t)\right|^{2}\right) .
\end{aligned}
$$

We can also calculate $\sigma_{\theta}^{2}$ as $\sigma_{\theta}^{2}=\left\langle\theta^{2}\right\rangle-\langle\theta\rangle^{2}$, where

$$
\begin{aligned}
& \langle\theta\rangle=\int_{0}^{\pi}\langle\theta, \phi=0 \mid \psi(t)\rangle\langle\psi(t)| \theta, \phi= \\
& 0\rangle \theta \sin (\theta) \mathrm{d} \theta=2 \pi \int_{0}^{\pi} Q(t) \theta \sin (\theta) \mathrm{d} \theta .
\end{aligned}
$$

For a simple case in Eq. (20) we take $(\varphi=0)$ so $\sigma_{\theta}^{2}$ can be written as

$$
\begin{aligned}
\sigma_{\theta}^{2} & =2 \pi \int_{0}^{\pi} Q(t) \theta^{2} \sin (\theta) \mathrm{d} \theta \\
& -\left[2 \pi \int_{0}^{\pi} Q(t) \theta \sin (\theta) \mathrm{d} \theta\right]^{2} .
\end{aligned}
$$

Using Eqs. (22) and (23) we can calculate integral in Eq. (20). Figure 4 shows the Fisher information as a function of time. Figure $4 \mathrm{a}$ is related to functions (4) and (5), Fig. $4 \mathrm{~b}$ to (6) and (7), Fig. $4 \mathrm{c}$ to (8) and (9). In Fig. 4a in some time durations entanglement is zero or system is separable, the length of these periods decreases with respect to time. For example the first time duration whose FI is zero, is between $t=3$ and $t=25$, second duration is $(t=40-55)$ and next period $(t=72$ $83)$ shows weak oscillations while the negativity and the concurrence (Figs. 2a and 3a) do not show this. We note that Fig. 4 shows curves with more oscillations than concurrence and negativity.

The curves appearance in Fig. 4b is not similar to that of Figs. 2b and 3b but their behavior is similar and the local maxima and minima have approximately the same feature. Of course, in Fig. 4b again the length of periods that system is separable reduces in far times. In Fig. 4c one can see that when the qubit interacting field is in squeezed state, the ESD phenomenon happens smoothly. Figure 5 shows our results for FI at far times $(g t<100)$, 


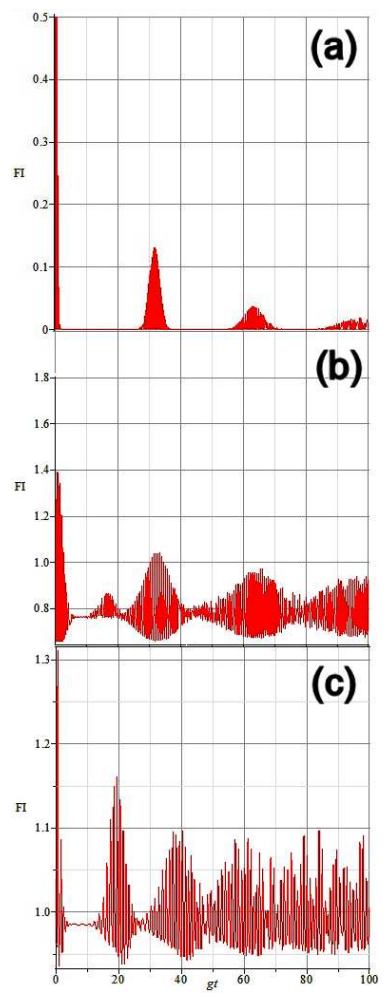

Fig. 4. The time evolution of the Fisher information for initial functions (a) (4) and (5), (b) (6) and (7), (c) (8) and (9) with $\lambda=0.5$.

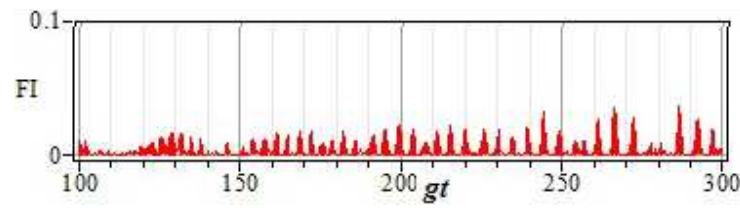

Fig. 5. The diagram of Fisher information in $t<100$.

we note that unlike concurrence and negativity the FI curves show small and weak oscillations at far times. In the other words, according to FI curves, two qubit entanglement do not die completely at far times but qubit is weakly entangled. Also qubit entanglement is strong if the interacting field changes from coherent state field to the squeezed state or the cat state.

The interesting observation here is that with increasing squeezing parameter $\lambda$, the ESD phenomenon does not happen for squeezed light but the qubit entanglement exhibits only a revival behavior (Fig. 6), so that the qubit entanglement falls abruptly and recover immediately and retains this behavior constantly. On the other words, the effect of squeezing light on two qubit entanglement is that in contrast the coherent field, the entanglement retains its revival (oscillating) behavior permanently and never dies completely. When we look at the curves of concurrence, negativity and Fisher information, we note that the Fisher information curves show more number of oscillations than curves of concurrence and negativity.

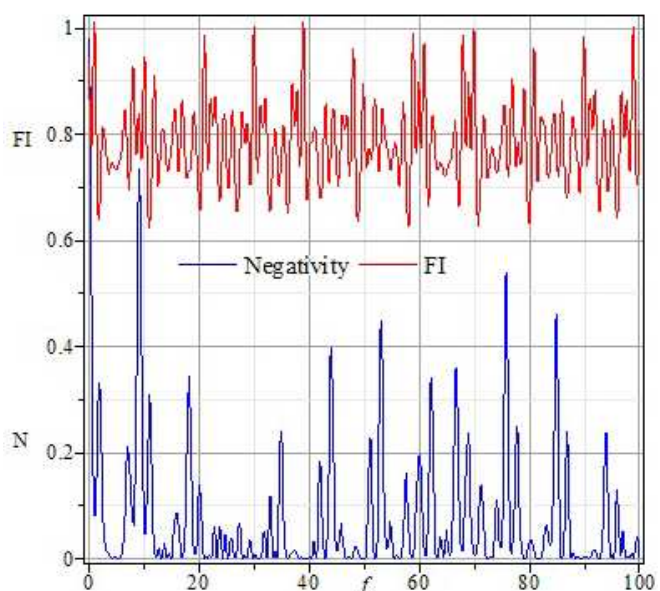

Fig. 6. The negativity and Fisher information for initial function (8) with $\lambda=1$.

\section{Conclusion}

In summary, we have investigated ESD phenomenon of two qubits with three different states of field, coherent state, cat state and squeezed state. We showed that for all three states of field, the entanglement exhibits damping oscillator behavior so that the entanglement occurrences are periodic in time but are not periodic in amplitude. We noted that if field is in the cat state or in the squeezed state, two-qubit entanglement decays slowly. The interesting observation here is that with increasing squeezing parameter $\lambda$, the entanglement falls suddenly and recovers immediately and retains its collapse-revival behavior permanently and never dies completely, this property is absent for the coherent state and the cat state of field. So, not only the infinite range of photon numbers effects on decoherence of system [12], but also with changing the nature of field we can control ESD. With selecting the coherent state as field, the entanglement deteriorates in finite time, with changing field to cat state this deterioration will happen slowly while the collapse-revival behavior events with more oscillation and entanglement of system maintains in more time durations than coherent state. We noted that in the squeezed state field case (for large squeezing parameter $\lambda$ ), the entanglement maintains its collapse-revival behavior forever (Fig. 7). In Fig. 8 we compare the concurrence and Fisher information for initial function (4). Another point is that the evolution of entanglement degree depends on the initial atomic state of the system, for initial atomic state (2) more degree entanglement has reported than initial atomic state (3). In this paper, we used concurrence and negativity as entanglement measures. By comparing the results of concurrence and negativity as entanglement measures with quantum Fisher information it has been shown that FI is not a good entanglement measure. 


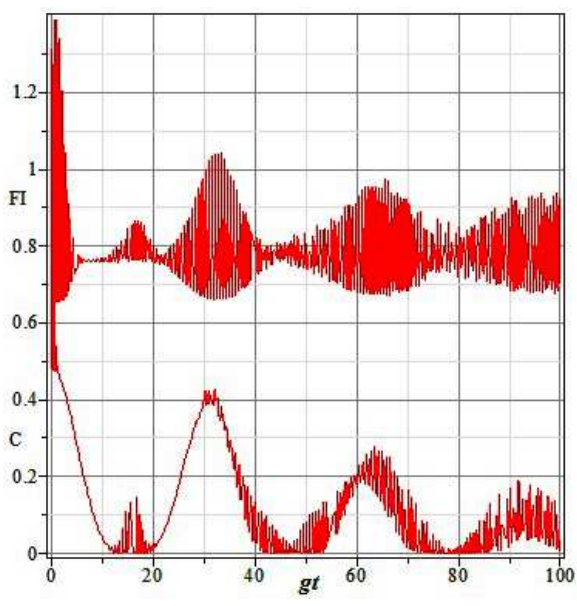

Fig. 7. Comparison between the negativity and Fisher information for initial function (6).

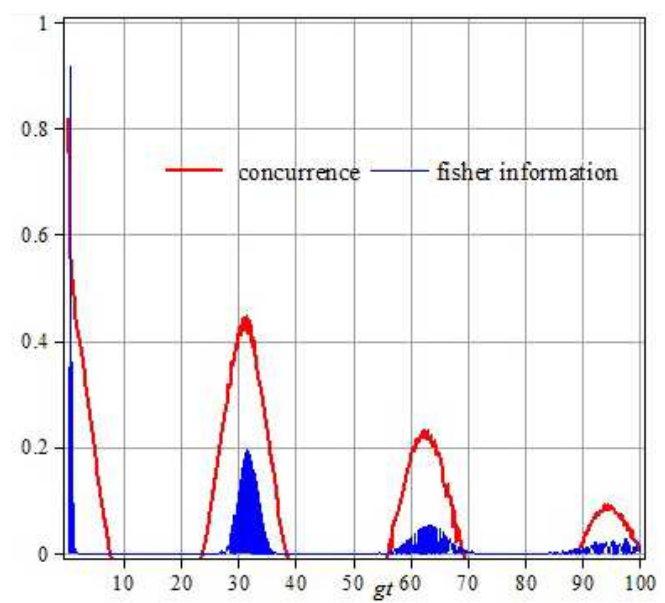

Fig. 8. Comparison between the concurrence and Fisher information for initial function (4).

\section{References}

[1] N. Gisin, G. Ribordy, W. Tittel, H. Zbinden, Rev. Mod. Phys. 74, 145 (2002).

[2] F.G. Deng, C.Y. Li, Y.S. Li, H.Y. Zhou, Y. Wang, Phys. Rev. A 72, 022338 (2005).

[3] F.G. Deng, G.L. Long, Phys. Rev. A 68, 042315 (2003).

[4] L. Xiao, G.L. Long, F.G. Deng, J.W. Pan, Phys. Rev. A 69, 052307 (2004).

[5] F.G. Deng, X.H. Li, C.Y. Li, P. Zhou, H.Y. Zhou, Phys. Rev. A 72, 044301 (2005).

[6] G.L. Long, X.S. Liu, Phys. Rev. A 65, 032302 (2002).
[7] C. Wang, F.G. Deng, Y.S. Li, X.S. Liu, G.L. Long, Phys. Rev. A 71, 044305 (2005).

[8] E.T. Jaynes, F.W. Cummings, Proc. IEEE 51, 89 (1963).

[9] T. Yu, J.H. Eberly, Phys. Rev. Lett. 93, 140404 (2004).

[10] T. Yu, J.H. Eberly, Opt. Commun. 264, 2 (2006).

[11] T. Yu, J.H. Eberly, Science 323, 598 (2009).

[12] M. Yönaç, T. Yu, J.H. Eberly, J. Phys. B At. Mol. Opt. Phys. 39, 621 (2006).

[13] M. Yönaç, J.H. Eberly, Opt. Lett. 33, 270 (2008).

[14] H.T. Cui, K. Li, X.X. Yi, Phys. Lett. A 365, 44 (2007).

[15] C.E. López, G. Romero, F. Lastra, E. Solano, J.C. Retamal, Phys. Rev. Lett. 101, 080503 (2008).

[16] F.F. Fanchini, P.E.M.F. Mendonca, R.D.J. Napolitano, Quant. Inform. Comput. 11, 0677 (2011).

[17] L. Mazzola, S. Maniscalo, J. Piilo, K.-A. Suominen, B.M. Garraway, Phys. Rev. A 79, 042302 (2009).

[18] X.-F. Qian, J.H. Eberly, Phys. Lett. A 376, 2931 (2012).

[19] M. Yönaç, J.H. Eberly, arXiv:1211.5654v1 (2012).

[20] H. Heydari, D. Hoang, AIP Conf. Proc. 1508, 391 (2012).

[21] G.F. Zhang, X.C. Xie, Europ. Phys. J. D 60, 423 (2010).

[22] J.G.G. de Oliveira, J.G. Peixoto de Faria, M.C. Nemes, Phys. Lett. A 375, 4255 (2011).

[23] T. Subeesh, V. Sudhir, A.B.M. Ahmed, M. Venkata Satyanarayana, Nonlin. Opt. Quantum Opt. Concepts Mod. Opt. 44, 245 (2012).

[24] C. Gerry, P. Knight, Introductory Quantum Optics, Cambridge University Press, Cambridge 2005.

[25] W.K. Wootters, Phys. Rev. Lett. 80, 2245 (1998).

[26] A. Peres, Phys. Rev. Lett. 77, 1413 (1996).

[27] M. Horodecki, P. Horodecki, R. Horodecki, Phys. Lett. A 223, 1 (1996).

[28] G. Vidal, R.F. Werner, Phys. Rev. A 65, 032314 (2002).

[29] M. Zhong-Xiao, X. Yun-Jie, B.A. Nguyen, J. Phys. B At. Mol. Opt. Phys. 41, 155501 (2008).

[30] R.A. Fisher, Proc. Cambridge Philos. Soc. 22, 700 (1925).

[31] A. Kitagawa, M. Takeoka, M. Sasaki, A. Chefles, arXiv:quant-ph/0612099v1 (2006).

[32] A.S.F. Obadaa, S. Abdel-Khalek, Physica A 389, 891 (2010).

[33] P. Hyllus, W. Laskowski, R. Krischek, C. Schwemmer, W. Wieczorek, H. Weinfurter, L. Pezzé, Phys. Rev. A 85, 022321 (2012). 